Tohoku Math. J.

54 (2002), 145-159

\title{
DEFORMATION AND STABILITY OF SURFACES WITH CONSTANT MEAN CURVATURE
}

\author{
MIYUKI KOISO
}

(Received April 26, 2000, revised February 13, 2001)

\begin{abstract}
For a CMC immersion from a two-dimensional compact smooth manifold with boundary into the Euclidean three-space, we give sufficient conditions under which it has a CMC deformation fixing the boundary. Moreover, we give a criterion of the stability for $\mathrm{CMC}$ immersions. Both of these are achieved by using the properties of eigenvalues and eigenfunctions of an eigenvalue problem associated to the second variation of the area functional. In a certain special case, by combining these results, we obtain a 'visible' way of judging the stability.
\end{abstract}

1. Introduction. An immersion $\mathcal{X}: M \rightarrow \boldsymbol{R}^{3}$ of a two-dimensional orientable compact connected $C^{\infty}$ manifold $M$ with boundary $\partial M$ into the Euclidean three-space $\boldsymbol{R}^{3}$ has constant mean curvature if and only if $\mathcal{X}$ is a critical point of the area functional for all volumepreserving variations of $\mathcal{X}$ that fix the boundary (cf. Barbosa-do Carmo [1, Proposition 2.7]). When the mean curvature of $\mathcal{X}$ is constant (we will say that $\mathcal{X}$ is a CMC immersion), $\mathcal{X}$ is said to be stable if the second variation of the area functional is nonnegative for all such variations of $\mathcal{X}$ as above.

The objective of this paper includes two themes. One of them is on the possibility of (not necessarily isometric) CMC deformation of CMC immersions that fix the boundary (Theorems 1.1 and 1.2). The other is on the determination of the stability or the unstability of CMC immersions (Theorem 1.3, Corollary 1.1). These two themes are related to each other in the following sense. First, both of these are achieved by using the properties of eigenvalues and eigenfunctions of an eigenvalue problem associated to the second variation of the area functional (Theorems 1.1, 1.2, and 1.3). Second, in the most characteristic case as a solution of a certain variational problem with constraint, a criterion of the stability of a CMC immersion is represented by the property of its CMC deformation fixing the boundary (Corollary 1.1).

Given a CMC immersion $\mathcal{X}: M \rightarrow \boldsymbol{R}^{3}$, consider a volume-preserving variation $\mathcal{X}_{t}$ of $\mathcal{X}$ that fixes the boundary. Denote by $A(t)$ the area of $\mathcal{X}_{t}$. Then

$$
A^{\prime \prime}(0)=-\int_{M}\left(\Delta u+\|B\|^{2} u\right) u d \omega,
$$

2000 Mathematics Subject Classification. Primary 58E12; Secondary 53C42, 53A10.

Partially supported by Grant-in-Aid for Scientific Research (C) No. 11640200 of the Japan Society for the Promotion of Science. 
where $u$ is the normal component of the variation vector field of $\mathcal{X}_{t}, \Delta=\operatorname{div}$ grad is the Laplacian on $M$ induced by $\mathcal{X},\|B\|^{2}$ is the squared norm of the second fundamental form of $\mathcal{X}$, and $d \omega$ is the area element of $M$ induced by $\mathcal{X}$.

Let us define a linear operator $L: H_{0}^{1}(M) \rightarrow L^{2}(M)$ (as for the definition of the function spaces $H_{0}^{1}(M)$ and $L^{2}(M)$, see Section 2) as

$$
L u=\Delta u+\|B\|^{2} u,
$$

and consider the eigenvalue problem

$$
\left\{\begin{array}{l}
L u=-\lambda u, \quad u \in H_{0}^{1}(M)-\{0\} . \\
\left.u\right|_{\partial M}=0,
\end{array}\right.
$$

Regarding the existence of CMC deformations, we will prove the following Theorems 1.1 and 1.2. Let $\alpha$ be an arbitrary fixed number such that $0<\alpha<1$.

THEOREM 1.1. Let $\mathcal{X} \in C^{3+\alpha}\left(M, \boldsymbol{R}^{3}\right)$ be an immersion with constant mean curvature $H_{0}$, and $\mathcal{N}: M \rightarrow S^{2}$ be its Gauss map. Assume that the eigenvalue problem (1.1) has no zero as its eigenvalue. Then there exist a neighborhood $W$ of $H_{0}$ in $\boldsymbol{R}$ and a unique injective $C^{1}$ mapping $\varphi: W \rightarrow C_{0}^{2+\alpha}(M)$ such that $\varphi\left(H_{0}\right)=0$ and that each

$$
\mathcal{X}+\varphi(H) \mathcal{N}, \quad H \in W
$$

is a $C^{2+\alpha}$ immersion of $M$ into $\boldsymbol{R}^{3}$ with constant mean curvature $H$. Moreover, in a small neighborhood of $\mathcal{X}$ in $C^{2+\alpha}\left(M, \boldsymbol{R}^{3}\right)$, there exists no other $C M C$ immersion (modulo $C^{2+\alpha}$ diffeomorphisms of $M$ ) with the same boundary value as $\mathcal{X}$.

When the problem (1.1) has zero eigenvalues, denote by $E$ the eigenspace of zero eigenvalues, and by $E^{\perp}$ its orthogonal complement in $L^{2}(M)$.

THEOREM 1.2. Let $\mathcal{X} \in C^{3+\alpha}\left(M, \boldsymbol{R}^{3}\right)$ be an immersion with constant mean curvature $H_{0}$, and $\mathcal{N}: M \rightarrow S^{2}$ be its Gauss map. Assume that $E$ is one-dimensional, and that $\int_{M}$ ed $\omega$ is not zero for any eigenfunction e in $E$. Then there exist a neighborhood $W$ of 0 in $E$ and a unique injective $C^{1}$ mapping $\psi=(\xi, \eta): W \rightarrow\left(C_{0}^{2+\alpha} \cap E^{\perp}\right) \times \boldsymbol{R}$, such that $\psi(0)=\left(0, H_{0}\right)$ and that each

$$
\mathcal{X}+(u+\xi(u)) \mathcal{N}, \quad u \in W
$$

is a $C^{2+\alpha}$ immersion of $M$ into $\boldsymbol{R}^{3}$ with constant mean curvature $\eta(u)$. Moreover, in a small neighborhood of $\mathcal{X}$ in $C^{2+\alpha}\left(M, \boldsymbol{R}^{3}\right)$, there exists no other $C M C$ immersion (modulo $C^{2+\alpha}$ diffeomorphisms of $M$ ) with the same boundary value as $\mathcal{X}$.

We remark that $\eta$ in Theorem 1.2 is not necessarily injective. In fact, each hemisphere is an example that $\eta$ is not injective.

Theorems 1.1 and 1.2 give deformations of CMC immersions with fixed boundary values, where the mean curvature continuously depends on each $\mathrm{CMC}$ immersion. On the other hand, Tomi [15] obtains deformations of disk-type CMC immersions with fixed mean curvature but with variable boundary values. 
Next, we turn our attention to the determination of the stability of CMC immersions. When we consider a variational problem without constraint, for example the case of minimal surfaces, the stability of a critical point is equivalent to the nonnegativity of the first eigenvalue of the eigenvalue problem associated to the second variation of the considered functional. However, for CMC immersions, the situation is different, and we can decide the stability by the following way: Let us denote by $\lambda_{i}=\lambda_{i}(M)$ the $i$-th eigenvalue of the problem (1.1).

THEOREM 1.3. Let $\mathcal{X} \in C^{3+\alpha}\left(M, \boldsymbol{R}^{3}\right)$ be an immersion with constant mean curvature.

(I) If $\lambda_{1} \geq 0$, then $\mathcal{X}$ is stable.

(II) If $\lambda_{1}<0<\lambda_{2}$, then there exists a uniquely determined function $u \in C_{0}^{3+\alpha}(M)$ which satisfies $L u=1$, and the following (II-1) and (II-2) hold:

(II-1) If $\int_{M} u d \omega \geq 0$, then $\mathcal{X}$ is stable.

(II-2) If $\int_{M} u d \omega<0$, then $\mathcal{X}$ is unstable.

(III) If $\lambda_{1}<0=\lambda_{2}$, then the following (III-A) and (III-B) hold:

(III-A) If there exists an eigenfunction g belonging to $\lambda_{2}$ which satisfies $\int_{M} g d \omega \neq$ 0 , then $\mathcal{X}$ is unstable.

(III-B) If $\int_{M} g d \omega=0$ for any eigenfunction g belonging to $\lambda_{2}$, then there exists a uniquely determined function $u \in E^{\perp} \cap C_{0}^{3+\alpha}(M)$ which satisfies $L u=1$, and the following (III-B1) and (III-B2) hold:

(III-B1) If $\int_{M} u d \omega \geq 0$, then $\mathcal{X}$ is stable.

(III-B2) If $\int_{M} u d \omega<0$, then $\mathcal{X}$ is unstable.

(IV) If $\lambda_{2}<0$, then $\mathcal{X}$ is unstable.

The positivity of the first eigenvalue and the negativity of the second eigenvalue of (1.1) are rather easily estimated by using the Gauss map of the considered CMC immersion (cf. Koiso [7, Corollary 1]). Therefore, (I) and (IV) of Theorem 1.3 are often useful. On the other hand, it is not easy to understand the geometric meaning of (II) and (III). However, for the case (II), by using Theorem 1.1, we can prove the following result which is easier to be seen.

COROLlary 1.1. Let $\mathcal{X} \in C^{3+\alpha}\left(M, \boldsymbol{R}^{3}\right)$ be an immersion with constant mean curvature. Assume that $\lambda_{1}<0<\lambda_{2}$. Then, in a small neighborhood $U$ of $\mathcal{X}$ in $C^{2+\alpha}\left(M, \boldsymbol{R}^{3}\right)$, there exists a unique (modulo $C^{2+\alpha}$ diffeomorphisms of $M$ ) one-parameter family $\left\{\mathcal{X}_{t}\right\}$ of $C M C$ immersions with the same boundary value as $\mathcal{X}$ such that the mean curvature $H(t)$ of $\mathcal{X}_{t}$ is a strictly monotone function of $t$. Parameter $t$ can be chosen so that $\mathcal{X}_{t}$ is of $C^{1}$ with respect to $t$ and that $H^{\prime}(0) \neq 0$. Denote by $V(t)$ the volume of $\mathcal{X}_{t}$. Then the following (1) and (2) hold:

(1) If $H^{\prime}(0) V^{\prime}(0) \geq 0$, then $\mathcal{X}$ is stable.

(2) If $H^{\prime}(0) V^{\prime}(0)<0$, then $\mathcal{X}$ is unstable.

In the case (III) of Theorem 1.3, consider an increasing sequence of closed domains $M_{s}$ of $M$ with smooth boundary, each of which is a proper subset of $M$ and converges to $M$ as $s \rightarrow \infty$. Then, by the monotonicity of eigenvalues (cf. Remark 2.1), any restriction $\left.\mathcal{X}\right|_{M_{s}}$ 
satisfies $\lambda_{1}<0<\lambda_{2}$ for large $s$. Therefore, we can apply Corollary 1.1 to each $\left.\mathcal{X}\right|_{M_{s}}$ and decide its stability. It is clear that if all such $\left.\mathcal{X}\right|_{M_{s}}$ are stable, then $\mathcal{X}$ is also stable, and if at least one such $\left.\mathcal{X}\right|_{M_{s}}$ is unstable, then so is $\mathcal{X}$.

We should mention works by Maddocks ([11], [12], [13]) and by Vogel ([16], [17], [18], [19]). Maddocks gave criteria of the stability for general variational problems with constraint. Our proofs of Theorem 1.3 (II) and Corollary 1.1 are essentially in line with Maddocks' methods. Vogel obtained results for CMC embeddings of revolution with free boundary on two parallel planes ([16]), for a general variational problem with constraint ([17]), and for capillary surfaces ([19]), similar to or more stringent than (I), (II), and (IV) of our Theorem 1.3. Moreover, [16] gave a result on CMC embeddings of revolution with free boundary on two parallel planes similar to our Corollary 1.1 .

This paper consists of five sections. Section 2 is a preparatory section, where we will give some fundamental definitions and notation, and recall basic properties of the eigenvalue problem (1.1). Section 3 will be devoted to proofs of Theorems 1.1 and 1.2. Theorem 1.3 and Corollary 1.1 will be proved in Section 4. Finally, in Section 5, we will give some examples and remarks in relation to our main results.

Before ending this section, we would like to propose a conjecture.

CONJECTURE 1.1. If $\mathcal{X}$ is a stable CMC immersion, then there exists a CMC deformation of $\mathcal{X}$ that fixes the boundary.

2. Preliminaries. Let $\mathcal{X}: M \rightarrow \boldsymbol{R}^{3}$ be a $C^{3+\alpha}$ immersion and $\mathcal{N}: M \rightarrow S^{2}$ be the Gauss map of $\mathcal{X}$. Then the area $A(\mathcal{X})$ and the volume $V(\mathcal{X})$ of $\mathcal{X}$ are defined as follows:

$$
A(\mathcal{X})=\int_{M} d \omega, \quad V(\mathcal{X})=\frac{1}{3} \int_{M}\langle\mathcal{X}, \mathcal{N}\rangle d \omega,
$$

where $\langle$,$\rangle is the usual inner product in \boldsymbol{R}^{3}$.

A one-parameter family $\left\{\mathcal{X}_{t}\right\}_{t \in(-\delta, \delta)}(\delta>0)$ of $C^{2+\alpha}$ immersions $\mathcal{X}_{t}: M \rightarrow \boldsymbol{R}^{3}$ is called a variation of $\mathcal{X}$, if $\mathcal{X}_{0}=\mathcal{X}$ and if $\mathcal{X}_{t}$ is of $C^{1}$ with respect to $t$. For convenience, we often write $\mathcal{X}_{t}$ instead of $\left\{\mathcal{X}_{t}\right\}_{t \in(-\delta, \delta)}$. $\partial \mathcal{X}_{t} /\left.\partial t\right|_{t=0}$ is called the variation vector field of $\mathcal{X}_{t}$.

For a variation $\mathcal{X}_{t}$ of $\mathcal{X}$, set $A(t)=A\left(\mathcal{X}_{t}\right)$ and $V(t)=V\left(\mathcal{X}_{t}\right)$. We will say that a variation $\mathcal{X}_{t}$ is volume-preserving if $V(t)=V(0)$ for all $t \in(-\delta, \delta)$, and that a variation $\mathcal{X}_{t}$ fixes the boundary if $\left.\mathcal{X}_{t}\right|_{\partial M}=\left.\mathcal{X}\right|_{\partial M}$ for all $t \in(-\delta, \delta)$.

Let $\mathcal{X}_{t}$ be a variation that fixes the boundary. Denote by $H_{t}$ the mean curvature of $\mathcal{X}_{t}$, by $\mathcal{N}_{t}$ the Gauss map of $\mathcal{X}_{t}$, and by $d \omega_{t}$ the volume element of $M$ relative to the metric induced by $\mathcal{X}_{t}$. Set

$$
u_{t}(z)=\left\langle\frac{\partial \mathcal{X}_{t}(z)}{\partial t}, \mathcal{N}_{t}(z)\right\rangle
$$

The following first variation formulas are well-known:

$$
A^{\prime}(t)=-2 \int_{M} H_{t} u_{t} d \omega_{t}, \quad V^{\prime}(t)=\int_{M} u_{t} d \omega_{t} .
$$


From now on, we assume that $\mathcal{X}: M \rightarrow \boldsymbol{R}^{3}$ is a CMC immersion. We would like to consider all $C^{2+\alpha}$ immersions $\mathcal{Y}: M \rightarrow \boldsymbol{R}^{3}$ which satisfy $\left.\mathcal{Y}\right|_{\partial M}=\left.\mathcal{X}\right|_{\partial M}$. The following Lemma can be observed in view of the discussion in Böhme and Tomi [2, §3].

LEMma 2.1. Let $\mathcal{X}: M \rightarrow \boldsymbol{R}^{3}$ be a $C^{3+\alpha}$ immersion. Then there exists a neighborhood $U$ of $\mathcal{X}$ in $C^{2+\alpha}\left(M, \boldsymbol{R}^{3}\right)$ such that the following statement is true: For each $\mathcal{Y} \in U$ satisfying $\left.\mathcal{Y}\right|_{\partial M}=\left.\mathcal{X}\right|_{\partial M}$, there exist a unique $C^{2+\alpha}$ diffeomorphism $\tau: M \rightarrow M$ and a unique function $u \in C_{0}^{2+\alpha}(M)$ such that

$$
\mathcal{Y} \circ \tau=\mathcal{X}+u \mathcal{N}
$$

Therefore, near $\mathcal{X}$, it is sufficient to consider only immersions represented as

$$
\mathcal{X}+u \mathcal{N}, \quad u \in C_{0}^{2+\alpha}(M),
$$

and hence, it is sufficient to consider only normal variations. For a normal variation $\mathcal{X}_{t}$, the following variation formula of the corresponding mean curvature $H_{t}$ is well-known (cf. Duschek [3], Kapouleas [6]).

$$
\left.2 \frac{\partial H_{t}}{\partial t}\right|_{t=0}=\Delta u_{0}+\|B\|^{2} u_{0} .
$$

Moreover, if $\mathcal{X}_{t}$ is volume-preserving and fixes the boundary, then we have

$$
A^{\prime \prime}(0)=-\int_{M}\left(\Delta u_{0}+\|B\|^{2} u_{0}\right) u_{0} d \omega .
$$

Definition 2.1. Let $\mathcal{X}: M \rightarrow \boldsymbol{R}^{3}$ be a CMC immersion. Then $\mathcal{X}$ is said to be stable if

$$
A^{\prime \prime}(0) \geq 0
$$

for all volume-preserving variations $\mathcal{X}_{t}$ of $\mathcal{X}$ which fix the boundary. When $\mathcal{X}$ is not stable, it is said to be unstable.

The following necessary and sufficient condition for the stability is known.

Theorem A (Barbosa and do Carmo [1, Proposition 2.10]). Let $\mathcal{X}$ be a CMC immersion. Then $\mathcal{X}$ is stable if and only if

$$
I(u):=-\int_{M}\left(\Delta u+\|B\|^{2} u\right) u d \omega \geq 0
$$

for all function $\mathrm{u}$ in

$$
\mathcal{F}:=\left\{u \in C_{0}^{2+\alpha}(M) \mid \int_{M} u d \omega=0\right\},
$$

where $C_{0}^{2+\alpha}(M)$ is the set of all real-valued functions on $M$ which are of $C^{2+\alpha}$ on $M$ and vanish on $\partial M$. 
Let us define some function spaces associated with $\mathcal{X}$. Denote by $L^{2}(M)$ the usual Hilbert space completion of $C^{\infty}(M)$ with respect to the norm defined by the inner product

$$
(u, v)_{L^{2}}=\int_{M} u v d \omega .
$$

We denote by $H_{0}^{1}(M)$ the completion of $C_{0}^{\infty}(M)$ with respect to the norm defined by the inner product

$$
(u, v)_{H^{1}}=\int_{M}(u v+\nabla u \nabla v) d \omega,
$$

where $\nabla u \nabla v$ denotes the inner product of the gradient of $u$ and that of $v$ with respect to the Riemannian metric of $M$ induced by $\mathcal{X}$. Let us denote by $\hat{H}(M)$ the pre-Hilbert space $H_{0}^{1}(M)$ with inner product $(,)_{L^{2}}$.

Now, we consider the linear operator $L: H_{0}^{1}(M) \rightarrow L^{2}(M)$ and the eigenvalue problem (1.1) both of which were defined in Section 1. When we consider $L$ as an operator from $C_{0}^{3+\alpha}(M)$ into $C^{1+\alpha}(M), L$ is strongly elliptic and self-adjoint with respect to the inner prod$\operatorname{uct}(,)_{L^{2}}$.

REMARK 2.1. The eigenvalue problem (1.1) has countably many eigenvalues (Smale [14, Lemma 1]):

$$
\lambda_{1}<\lambda_{2} \leq \cdots, \quad \lambda_{n} \rightarrow+\infty(n \rightarrow+\infty) .
$$

Each eigenfunction of (1.1) is of $C_{0}^{3+\alpha}(M)$ (cf. Gilbarg-Trudinger [4, Theorem 8.13] and Ladyzhenskaya-Ural'tseva [9, Chap. 3, Theorem 12.1]). Denote by $\varphi_{i}$ an eigenfunction of (1.1) belonging to $\lambda_{i}$. $\left\{\varphi_{i}\right\}$ can be chosen so that they form an orthonormal basis for $L^{2}(M)$ (Smale [14, Lemma 2]). Moreover, each $\lambda_{i}$ has the following minimum property on the preHilbert space $\hat{H}(M)$ (Smale [14, Lemma 4]).

$$
\begin{aligned}
& \lambda_{1}=I\left(\varphi_{1}\right)=\min \left\{I(u) \mid u \in H_{0}^{1}(M) \text { and } \int_{M} u^{2} d \omega=1\right\}, \\
& \lambda_{i}=I\left(\varphi_{i}\right)=\min \left\{I(u) \mid u \in H_{0}^{1}(M), \int_{M} u^{2} d \omega=1\right. \text { and } \\
& \left.\qquad \int_{M} u \varphi_{j} d \omega=0 \text { for } j \in\{1, \ldots, i-1\}\right\}, \quad i=2,3, \ldots .
\end{aligned}
$$

Also, the monotonicity of eigenvalues is verified (cf. Smale [14, Lemma 2]) by virtue of the property that $L$ has uniqueness in the Cauchy problem (cf. Hörmander [5, Chap. VIII]), that is, if $M_{1}$ is a proper subset of $M$ such that $\partial M_{1}$ is of $C^{3+\alpha}$, then $\lambda_{i}\left(M_{1}\right)>\lambda_{i}(M)$.

By using the Riesz-Schauder alternative theorem combined with the regularity theorem for solutions of strictly elliptic partial differential equations and the regularity of the CMC immersion $\mathcal{X}$, we obtain the following

LEMMA 2.2. Let $\lambda$ be a real number. We assume that $\partial M \in C^{k+2+\alpha}(k \in Z, k \geq$ $0,0<\alpha<1)$. 
(a) Assume that $\lambda$ is not an eigenvalue of (1.1). Then, for any function $f \in L^{2}(M)$, the equation

$$
\lambda u-L u=f
$$

has a uniquely determined solution $u \in H_{0}^{1}(M)$. Moreover, if $f \in C^{k+\alpha}(M)$, then the solution $u$ is in $C_{0}^{k+2+\alpha}(M)$.

(b) Assume that $\lambda$ is an eigenvalue of (1.1). Then, for each function $f \in L^{2}(M)$, the equation

$$
\lambda u-L u=f
$$

has a solution $u \in H_{0}^{1}(M)$ if and only if

$$
\int_{M} f v d \omega=0
$$

for all eigenfunctions $v$ belonging to $\lambda$. Moreover, if $f \in C^{k+\alpha}(M)$, then the solution $u$ is in $C_{0}^{k+2+\alpha}(M)$.

3. Proofs of Theorems 1.1 and 1.2. Let us assume that $\mathcal{X}: M \rightarrow \boldsymbol{R}^{3}$ is a $C^{3+\alpha}$ immersion with constant mean curvature $H_{0}$. Choose a neighborhood $V$ of 0 in $C_{0}^{2+\alpha}(M)$ so that, for any $u \in V, \mathcal{X}+u \mathcal{N}: M \rightarrow \boldsymbol{R}^{3}$ is an immersion. For any $u \in V$, denote by $H_{u}$ the mean curvature of $\mathcal{X}+u \mathcal{N}$.

Define a mapping

$$
\Phi: V \times \boldsymbol{R} \rightarrow C^{\alpha}(M)
$$

by

$$
\Phi(u, H)=2\left(H-H_{u}\right) .
$$

Then we first note the following

CLAIM 3.1.

$$
\Phi\left(0, H_{0}\right)=0 .
$$

The mean curvature of $\mathcal{X}+u \mathcal{N}(u \in V)$ is constant if and only if

$$
\Phi(u, H)=0
$$

for some $H \in \boldsymbol{R}$.

Lemma 3.1. $\Phi(u, H)$ is Fréchet differentiable with respect to $u$ and $H$, and

$$
\begin{aligned}
& D_{u} \Phi(0, H) v=-\left(\Delta v+\|B\|^{2} v\right), \quad H \in \boldsymbol{R}, \quad v \in C_{0}^{2+\alpha}(M), \\
& D_{H} \Phi(u, H) K=2 K, \quad H, K \in \boldsymbol{R}, \\
& D_{(u, H)} \Phi(0, H)(v, K)=2 K-\left(\Delta v+\|B\|^{2} v\right), \\
& H \in \boldsymbol{R}, \quad(v, K) \in C_{0}^{2+\alpha}(M) \times \boldsymbol{R} .
\end{aligned}
$$


ProOF. Since

$$
\lim _{t \rightarrow 0} \frac{\Phi(u+t v, H)-\Phi(u, H)}{t}=-\left.2 \frac{\partial H_{u+t v}}{\partial t}\right|_{t=0},
$$

the Gâteau derivative of $\Phi(u, H)$ with respect to $u$ is

$$
-\left.2 \frac{\partial H_{u+t v}}{\partial t}\right|_{t=0},
$$

and this operator is continuous in $v$. Therefore, $\Phi(u, H)$ is Fréchet differentiable with respect to $u$, and

$$
D_{u} \Phi(0, H) v=-\left.2 \frac{\partial H_{t v}}{\partial t}\right|_{t=0}=-\left(\Delta v+\|B\|^{2} v\right),
$$

where we used (2.2), and hence we have proved (3.3).

Since $\Phi(u, H)$ is linear with respect to $H$, the Fréchet differentiability with respect to $H$ and the formula (3.4) are clearly valid. (3.5) is obtained from (3.3) and (3.4).

LEMMA 3.2. Assume that zero is not an eigenvalue of the problem (1.1). Then the linear operator

$$
D_{u} \Phi\left(0, H_{0}\right): C_{0}^{2+\alpha}(M) \rightarrow C^{\alpha}(M)
$$

is bijective.

Proof. The injectivity is clear by (3.3). The surjectivity is obtained from Lemma 2.2(a).

Now we are ready to prove Theorem 1.1.

Proof of TheOrem 1.1. By virtue of Lemma 3.2, we can apply the implicit mapping theorem (cf. Lang [10, pp. 17-18]) to the mapping

$$
\Phi: V \times \boldsymbol{R} \rightarrow C^{\alpha}(M) .
$$

In view of Lemma 2.1 and Claim 3.1, we obtain Theorem 1.1.

Now, let us consider the case where the eigenvalue problem (1.1) has zero eigenvalues. As in Section 1, we denote by $E$ the eigenspace corresponding to zero eigenvalues, and by $E^{\perp}$ its orthogonal complement in $L^{2}(M)$. Define a mapping

$$
\Psi:(V \cap E) \times\left(V \cap E^{\perp}\right) \times \boldsymbol{R} \rightarrow C^{\alpha}(M)
$$

by

$$
\Psi(u, v, H)=\Phi(u+v, H) .
$$

Then, in view of Lemma 3.1, $\Psi(u, v, H)$ is Fréchet differentiable with respect to $u$, $v$, and $H$, and we have

$$
D_{(v, H)} \Psi\left(0,0, H_{0}\right)(w, H)=2 H-\left(\Delta w+\|B\|^{2} w\right), \quad(w, H) \in\left(C_{0}^{2+\alpha} \cap E^{\perp}\right) \times \boldsymbol{R} .
$$

LeMma 3.3. Assume that

$$
\operatorname{dim} E=1
$$


and that

$$
\int_{M} e d \omega \neq 0 \text { for some (and therefore all) } e \in E-\{0\} .
$$

Then the linear operator

$$
D_{(v, H)} \Psi\left(0,0, H_{0}\right):\left(C_{0}^{2+\alpha}(M) \cap E^{\perp}\right) \times \boldsymbol{R} \rightarrow C^{\alpha}(M)
$$

is bijective.

ProOF. By assumption, $E$ is represented as

$$
E=\left\{a e_{0} \mid a \in \boldsymbol{R}\right\},
$$

where $e_{0}$ is an eigenfunction with eigenvalue zero satisfying

$$
\int_{M} e_{0} d \omega \neq 0
$$

Set

$$
F=D_{(v, H)} \Psi\left(0,0, H_{0}\right)
$$

In view of (3.7), we see

$$
\operatorname{Ker} F=\left\{(w, H) \in\left(C_{0}^{2+\alpha} \cap E^{\perp}\right) \times \boldsymbol{R} \mid 2 H-\left(\Delta w+\|B\|^{2} w\right)=0\right\} .
$$

Suppose that $(w, H) \in\left(C_{0}^{2+\alpha} \cap E^{\perp}\right) \times \boldsymbol{R}$ is an element of $\operatorname{Ker} F$. Then

$$
-L w=-2 H \text {. }
$$

Hence, by Lemma 2.2(b),

From (3.9), we obtain

$$
-2 H \int_{M} e_{0} d \omega=0
$$

$$
H=0 \text {. }
$$

Therefore, by (3.10), $w$ is an element of $E$. On the other hand, by assumption, $w$ is in $E^{\perp}$. Hence

and we have proved

$$
w=0
$$

$$
\operatorname{Ker} F=\{(0,0)\}
$$

which implies the injectivity of $F$.

Now let us prove the surjectivity of $F$. For any function $f \in C^{\alpha}(M)$, set

$$
H=\frac{\int_{M} f e_{0} d \omega}{2 \int_{M} e_{0} d \omega} .
$$

Then, by Lemma 2.2(b), there exists a function $u \in C_{0}^{2+\alpha}(M)$ which satisfies

$$
-L u=f-2 H \text {. }
$$

Let us decompose $u$ into

$$
u=e+w, \quad e \in E, w \in E^{\perp}
$$


Then $w \in C_{0}^{2+\alpha}(M) \cap E^{\perp}$, and

$$
-L w=-L u=f-2 H,
$$

which implies that

$$
F(w, H)=f, \quad(w, H) \in\left(C_{0}^{2+\alpha}(M) \cap E^{\perp}\right) \times \boldsymbol{R} .
$$

Now, we are in the last stage of proving Theorem 1.2.

Proof of TheOrem 1.2. By virtue of Lemma 3.3, we can apply the implicit mapping theorem (cf. Lang [10, pp. 17-18]) to the mapping

$$
\Psi:(V \cap E) \times\left(V \cap E^{\perp}\right) \times \boldsymbol{R} \rightarrow C^{\alpha}(M) .
$$

Remark that, by Lemma 2.2(b) (or Remark 2.1), $E \subset C_{0}^{3+\alpha}(M) \subset C_{0}^{2+\alpha}(M)$. In view of Lemma 2.1 and Claim 3.1, we obtain Theorem 1.2.

\section{Proofs of Theorem 1.3 and Corollary 1.1 .}

Proof of TheOREM 1.3. (I) is clear from (2.3).

Assume that $\lambda_{1}<0$. As in Remark 2.1, let $\varphi_{i}$ be the eigenfunction corresponding to the $i$-th eigenvalue $\lambda_{i}$ of (1.1) such that $\left\{\varphi_{i}\right\}$ form an orthonormal basis for $L^{2}(M)$. Since $\varphi_{1}$ does not change sign,

$$
\int_{M} \varphi_{1} d \omega \neq 0
$$

For a function $u \in C_{0}^{3+\alpha}(M)$, set

$$
v=-\frac{\int_{M} u d \omega}{\int_{M} \varphi_{1} d \omega} \varphi_{1}+u .
$$

Then

$$
\int_{M} v d \omega=0 \text {. }
$$

By setting

$$
a=-\frac{\int_{M} u d \omega}{\int_{M} \varphi_{1} d \omega}
$$

$v$ is represented as

$$
v=a \varphi_{1}+u
$$

When (1.1) has no zero eigenvalue, by virtue of Lemma 2.2(a) and Remark 2.1, there exists a unique function $u \in C_{0}^{3+\alpha}(M)$ satisfying $L u=1$. In this case, by using the Green's 
formula and (4.3), we see that

$$
\begin{aligned}
I(v) & =-\int_{M} v L v d \omega \\
& =-\int_{M}\left(a^{2} \varphi_{1} L \varphi_{1}+a \varphi_{1} L u+a u L \varphi_{1}+u L u\right) d \omega \\
& =a^{2} \lambda_{1} \int_{M} \varphi_{1}^{2} d \omega-2 a \int_{M} \varphi_{1} L u d \omega-\int_{M} u d \omega \\
& =a^{2} \lambda_{1}+\int_{M} u d \omega .
\end{aligned}
$$

Since $\lambda_{1}<0, I(v)<0$ in the case where $\int_{M} u d \omega<0$. In this case, in view of Theorem A, $\mathcal{X}$ is unstable, which proves (II-2).

Next, when $u=\varphi_{2}$, from (4.4) and the orthonormality of $\left\{\varphi_{i}\right\}$, we see

$$
\begin{aligned}
I(v) & =a^{2} \lambda_{1} \int_{M} \varphi_{1}^{2} d \omega-2 a \int_{M} \varphi_{1} L \varphi_{2} d \omega-\int_{M} \varphi_{2} L \varphi_{2} d \omega \\
& =a^{2} \lambda_{1} \int_{M} \varphi_{1}^{2} d \omega+2 a \lambda_{2} \int_{M} \varphi_{1} \varphi_{2} d \omega+\lambda_{2} \int_{M} \varphi_{2}^{2} d \omega \\
& =\lambda_{1}\left(\int_{M} \varphi_{2} d \omega\right)^{2}\left(\int_{M} \varphi_{1} d \omega\right)^{-2}+\lambda_{2} .
\end{aligned}
$$

Therefore, if $\lambda_{2} \leq 0$ and $\int_{M} \varphi_{2} \neq 0$, then $I(v)<0$, which proves (III-A). When $\lambda_{2}<0$, also $I(v)<0$, which proves (IV).

Next we prove (II-1). Set

$$
E_{1}=\left\{a \varphi_{1} \mid a \in \boldsymbol{R}\right\}, \quad E_{1}^{\perp}=\left\{u \in C_{0}^{3+\alpha}(M) \mid \int_{M} \varphi_{1} u d \omega=0\right\} .
$$

By virtue of Lemma 2.2(a) and Remark 2.1, there exists a unique function $u \in C_{0}^{3+\alpha}(M)$ satisfying $L u=1$. If

$$
\int_{M} u d \omega \geq 0
$$

then

$$
I(u)=-\int_{M} u L u d \omega=-\int_{M} u d \omega \leq 0 .
$$

(2.4), (4.5) and $\lambda_{2}>0$ imply $u \notin E_{1}^{\perp}$. Therefore, any $v \in \mathcal{F}$ is represented as follows:

$$
v=w+b u, \quad b \in \boldsymbol{R}, \quad w \in E_{1}^{\perp} .
$$


Then

$$
\begin{aligned}
I(v) & =-\int_{M}\left(b^{2} u L u+b u L w+b w L u+w L w\right) d \omega \\
& =-b^{2} \int_{M} u d \omega-2 b \int_{M} w d \omega+I(w) \\
& =b^{2} \int_{M} u d \omega-2 b \int_{M}(w+b u) d \omega+I(w) \\
& =-b^{2} I(u)+I(w) \\
& \geq 0,
\end{aligned}
$$

which implies that $\mathcal{X}$ is stable.

Finally, we prove (III-B). By virtue of Lemma 2.2(b) and Remark 2.1, there exists a unique function $u \in E^{\perp} \cap C_{0}^{3+\alpha}(M)$ that satisfies $L u=1$.

When $\int_{M} u d \omega<0$, we can prove that $\mathcal{X}$ is unstable by the same way as in the proof of (II-1).

When $\int_{M} u d \omega>0$, we can prove that $\mathcal{X}$ is stable by the same way as in the proof of

When $\int_{M} u d \omega=0$,

$$
I(u)=-\int_{M} u L u d \omega=-\int_{M} u d \omega=0=\lambda_{2} .
$$

Assume that $u \in E_{1}^{\perp}$. By (2.4), $u$ is an eigenfunction corresponding to $\lambda_{2}=0$, that is, $L u=0$, which is a contradiction. Therefore, $u \notin E_{1}^{\perp}$, and hence, we can prove the stability of $\mathcal{X}$ by the same way as in the proof of (II- 1$)$.

Proof of COROLlary 1.1. By virtue of Theorem 1.1, the existence of a oneparameter family $\left\{\mathcal{X}_{t}\right\}$ of CMC immersions stated in the first half of Corollary 1.1 is verified.

From (2.2),

$$
2 H^{\prime}(0)=L u, \quad u=\left\langle\left.\frac{\partial \mathcal{X}_{t}}{\partial t}\right|_{t=0}, \mathcal{N}\right\rangle .
$$

On the other hand, from (2.1),

$$
V^{\prime}(0)=\int_{M} u d \omega .
$$

(1) and (2) are verified by (4.6), (4.7) and Theorem 1.3(II).

5. Examples and concluding remarks. In this section, we give some examples and remarks relating to our results which were obtained in the preceding sections.

Besides the plane, the simplest examples of CMC immersions are so-called Delaunay surfaces, which are rotational surfaces with constant mean curvature. Delaunay surfaces are classified into five classes, namely catenoids, spheres, cylinders, unduloids, and nodoids. Catenoids are only minimal surfaces among Delaunay surfaces. 
Let us consider connected compact subsets of Delaunay surfaces each of whose boundary (if it is not empty) consists of one or two circles that are perpendicular to the rotation axis. In the following, we will call each such subset to be an admissible piece. Any admissible piece with only one boundary component is a subset of a sphere, which is called a spherical cap. For an admissible piece $S$ of a Delaunay surface, we denote by $\lambda_{i}(S)$ the $i$-th eigenvalue of the eigenvalue problem (1.1) for $S$. This notation is reasonable, because the eigenvalues of (1.1) are independent of each parametrization of $\mathcal{X}(M)$ in the following sense: Let $\tau: M \rightarrow M$ be a $C^{3+\alpha}$ diffeomorphism of $M$, and set $\mathcal{Y}=\mathcal{X} \circ \tau$. Then the eigenvalues for $\mathcal{Y}$ are the same as those for $\mathcal{X}$.

First, let us consider an admissible piece $S$ of a catenoid $\hat{S}$. It is known that if the area of the image of the Gauss map of a minimal surface is smaller than $2 \pi$, then $\lambda_{1}>0$ (Barbosa-do Carmo [1]). Therefore, if $S$ is sufficiently small, then $\lambda_{1}$ is positive. Hence, by Theorem 1.1, there exists a one-parameter family $\left\{S_{t}\right\}_{t}\left(S_{0}=S\right)$ of embedded CMC surfaces from an annulus with the same boundary as $S$ such that the mean curvature $H(t)$ of $S_{t}$ is strictly monotone with respect to $t$. By using the Alexandrov reflection methods, each $S_{t}$ proves to be a surface of revolution. From the strict monotonicity of $H(t), S_{t}$ must be a part of an unduloid for $t>0$, and a part of a nodoid for $t<0$ (or, a part of an unduloid for $t<0$, and a part of a nodoid for $t>0$ ).

Next, we consider spherical caps. It is well-known that the first eigenvalue of the problem (1.1) for a hemisphere is zero, and the second eigenvalue for a whole sphere is zero as well. On the other hand, for each spherical cap $S$, it is easily observed that there exists a CMC deformation $\left\{S_{t}\right\}_{t}$ of $S$ such that $S_{0}=S$ and that each $S_{t}$ is a spherical cap with $\partial S_{t}=\partial S$. When $S$ is not a hemisphere, $\left\{S_{t}\right\}_{t}$ gives an example of Theorem 1.1. When $S$ is a hemisphere, $\left\{S_{t}\right\}_{t}$ gives an example of Theorem 1.2. When $S$ is larger than the hemisphere, $S$ gives an example of Corollary 1.1(1).

Next, let $\hat{S}$ be a cylinder of radius $r$. For each admissible piece $S$ of $\hat{S}$, we denote by $h=h(S)$ the distance between two boundary circles of $S$. The largest stable admissible piece is the one with $h=2 \pi r$, and in this case $\lambda_{2}(S)=0$. On the other hand, if $h=\pi r$, then $\lambda_{1}=0$. (cf. Barbosa-do Carmo [1, Example 2.17].) When $\pi r<h(S)<2 \pi r$, one can observe a smooth one-parameter family $\left\{S_{t}\right\}_{t}$ of admissible pieces of unduloids such that $S_{0}=S, \partial S_{t}=\partial S$, and $H^{\prime}(0) V^{\prime}(0)>0$. These observations correspond with Corollary $1.1(1)$.

Similar observations are done for unduloids. Hereafter, a circle of an unduloid (or a nodoid) $\hat{S}$ means a circle included in $\hat{S}$ which is perpendicular to the rotation axis of $\hat{S}$.

Let $\hat{S}$ be an unduloid. Among all admissible pieces of $\hat{S}$ one of whose boundary components is the largest (or smallest) circle of $\hat{S}$, the largest stable ones are those which correspond to exactly one period of $\hat{S}$ (Koiso [8, Chap. 5, §5.7]). In this case, $\lambda_{2}=0$. On the other hand, when $\partial S=C_{1} \cup C_{2}=$ (one of the largest circles of $\hat{S}$ ) $\cup$ (one of the nearest circles to $C_{1}$ among the smallest circles of $\hat{S}), \lambda_{1}(S)=0$. In fact, for any unit vector $v$ which is parallel to the rotation axis of $\hat{S}$, the function $u=\langle\mathcal{N}, v\rangle$ gives an eigenfunction corresponding to the 
zero eigenvalue, where $\mathcal{N}$ is the Gauss map of $S$, and zero is the first eigenvalue by virtue of the property that $u$ does not change sign in the interior of $S$.

Moreover, for an admissible piece $S$ with $\lambda_{1}(S)<0<\lambda_{2}(S)$, one can observe a smooth one-parameter family $\left\{S_{t}\right\}_{t}$ of admissible pieces of unduloids with $S_{0}=S$ and with $\partial S_{t}=\partial S$ such that $H^{\prime}(0) V^{\prime}(0)>0$.

Finally, let $\hat{S}$ be a nodoid. Denote by $\ell$ the rotation axis of $\hat{S}$. Let $S$ be an admissible piece of $\hat{S}$ with $\partial S=C_{1} \cup C_{2}$, where each $C_{i}$ is a circle in $\hat{S}$.

Assume first that, on $C_{1}$, the normal to $S$ is parallel to $\ell$. The largest stable such $S$ is the case where $C_{2}$ is one of the two neighboring circles on which the normal to $S$ is parallel to $\ell$, and in these cases, $\lambda_{2}(S)=0$ (Koiso [8, Chap. 5, §5.7]).

Assume next that $C_{1}$ is one of the smallest circles of $\hat{S}$. Denote by $C^{*}$ one of the nearest circles among the largest circles of $\hat{S}$, and denote by $S^{*}$ the admissible piece of $\hat{S}$ with $\partial S^{*}=$ $C_{1} \cup C^{*}$. Then $\lambda_{1}\left(S^{*}\right)=0$. Indeed, this is verified by the same way as for an admissible piece $S$ of an unduloid above, by considering function $u=\langle\mathcal{N}, v\rangle$, where $v$ is any unit vector which is parallel to $\ell$. Now, let $S$ be an admissible piece of $\hat{S}$ such that one of its boundary components is $C_{1}$ and that it contains $C^{*}$ in its interior. If $S$ is sufficiently small, then $\lambda_{1}(S)<0<\lambda_{2}(S)$. For such $S$, we conjecture the following.

CONJECTURE 5.1. There exists a smooth one-parameter family $\left\{S_{t}\right\}_{t}$ of admissible pieces of nodoids such that $S_{0}=S, \partial S_{t}=\partial S$, and $H^{\prime}(0) V^{\prime}(0)<0$.

If this conjecture is verified, then we obtain an example for which Corollary 1.1(2) can be applied, and we can conclude that $S$ is unstable.

\section{REFERENCES}

[ 1 ] J. L. BARboS A AND M. Do CARMo, Stability of hypersurfaces with constant mean curvature, Math. Z. 185 (1984), 339-353.

[ 2 ] R. Böhme AND F. Tomi, Zur Struktur der Lösungsmenge des Plateauproblems, Math. Z. 133 (1973), 1-29.

[ 3 ] A. DuscheK, Zur geometrischen Variationsrechnung, Math. Z. 40 (1936), 279-291.

[ 4 ] D. Gilbarg And N. S. Trudinger, Elliptic Partial Differential Equations of Second Order (Second Edition), Springer-Verlag, Berlin-Heidelberg-New York-Tokyo, 1983.

[ 5 ] L. HöRmander, Linear Partial Differential Operators, Third Revised Printing, Springer-Verlag, BerlinHeidelberg-New York, 1969.

[ 6 ] N. KAPOULEAS, Complete constant mean curvature surfaces in euclidean three-space, Ann. of Math. (2) 131 (1990), 239-330.

[ 7 ] M. KoISo, The stability and the vision number of surfaces with constant mean curvature, Bull. Kyoto Univ. Ed. Ser. B 92 (1998), 1-11.

[ 8 ] N. KoIso, Variational Problems (in Japanese), Kyōritu Publ., Tokyo, Japan, 1998.

[9] O. A. LADYZhenskaYa AND N. N. URAL'TSEVA, Linear and Quasilinear Elliptic Equations, Academic Press, New York and London, 1968.

[10] S. LANG, Differential Manifolds, Springer-Verlag, New York-Berlin-Heidelberg-Tokyo, 1985.

[11] J. H. MADDOCKS, Stability of nonlinearly elastic rods, Arch. Rat. Mech. Anal. 85 (1984), 311-354.

[12] J. H. MADDOCKS, Restricted quadratic forms and their application to bifurcation and stability in constrained variational principles, SIAM J. Math. Anal. 16 (1985), 47-68.

[13] J. H. MAdDOcKs, Stability and folds, Arch. Rat. Mech. Anal. 99 (1987), 301-328.

[14] S. SMALE, On the Morse index theorem, J. Math. Mech. 14 (1965), 1049-1055.

[15] F. TOMI, A perturbation theorem for surfaces of constant mean curvature, Math. Z. 141 (1975), 253-264. 
[16] T. I. VogeL, Stability of a liquid drop trapped between two parallel planes, SIAM J. Appl. Math. 147 (1987), 516-525.

[17] T. I. VogeL, On constrained extrema, Pacific J. Math. 176 (1996), 557-561.

[18] T. I. VogeL, Sufficient conditions for multiply constrained extrema, Pacific J. Math. 180 (1997), 377-383.

[19] T. I. VogEL, Non-linear stability of a certain capillary surfaces, Differential equations and dynamical systems (Waterloo, ON, 1997), Dynam. Contin. Discrete Impuls. Systems 5 (1999), 1-15.

DEPARTMENT OF MATHEMATICS

KYOTO UNIVERSITY OF EDUCATION

1 FUKAKUSA-FUJINOMORI-CHO

FUSHIMI-KU, КYOTO 612-8522

JAPAN

E-mail address: koiso@kyokyo-u.ac.jp 